\title{
Clinical benefit of fulvestrant monotherapy in the multimodal treatment of hormone receptor and HER2 positive advanced breast cancer:
}

\section{a case series}

This article was published in the following Dove Press journal:

OncoTargets and Therapy

Orsolya Rusz

Renáta Kószó

Ágnes Dobi

Melinda Csenki

Erzsébet Valicsek

Alíz Nikolényi

Gabriella Uhercsák

Adrienne Cserháti

Zsuzsanna Kahán

Department of Oncotherapy, University of Szeged, Szeged, Hungary

Correspondence: Zsuzsanna Kahán Department of Oncotherapy, University of Szeged, Korányi fasor 12, Szeged $\mathrm{H}-6720$, Hungary

Tel +36 62545404

Fax +3662545922

Email kahan.zsuzsanna@med.u-szeged.hu

\begin{abstract}
Fulvestrant is a pure estrogen receptor (ER) antagonist approved for the treatment of metastatic ER positive breast cancer in postmenopausal women with disease progression following antiestrogen therapy. The clinical results of fulvestrant demonstrated encouraging activity in tumors in spite of HER2 positivity, but data about its use after progression on antiHER2 agents are limited. Partial responses and durations of response of 12, 25, and 38 months in three cases with multiple metastases of ER positive and HER2 positive breast cancer were observed; all patients had been treated with 1-4 regimens of an anti-HER2 agent in combination with chemotherapy or an aromatase inhibitor before the initiation of fulvestrant. Fulvestrant is a valuable option with limited toxicity and durable response in metastatic HER2 and ER positive breast cancer after progression on anti-HER2 agents as well. Therapeutic benefit even in extensive skin metastases and (irradiated) brain metastases may be expected. Further investigations are warranted to establish where it fits into the multimodal management of ER and HER positive breast cancer.
\end{abstract}

Keywords: endocrine resistance, trastuzumab resistance, brain metastasis, skin metastasis

\section{Introduction}

Approximately $20 \%$ of advanced breast cancers overexpress HER 2 and approximately half of them also express hormone receptors (HRs). Within the HER2 positive disease entity, the HR positive status defines an individual subset with various clinical behaviors, which hinders the adjustment of optimal therapeutic strategy. ${ }^{1,2}$

Although the presence of HER2 and estrogen receptor (ER) predicts benefit from both HER2-targeted and endocrine therapy (ET), the simultaneous activity of the two pathways and their bidirectional cross-talk may result in de novo or acquired resistance to these agents. ${ }^{3,4}$ For the delay or prevention of the development of drug resistance, dual inhibition appears promising. ${ }^{2,5}$ Clinical trials that investigated firstline mono-aromatase inhibitor (mono-AI) treatment vs the combination with lapatinib or trastuzumab proved the superior effect of the combination, nonetheless, with no benefit in overall survival. ${ }^{6-9}$ Since, no studies have directly compared ET plus HER2targeted therapy vs chemotherapy plus HER2-targeted therapy; chemotherapy plus trastuzumab is still the preferred choice in HER2 and HR positive cases. ${ }^{10}$ With the aim of long-term control of advanced HER2 and HR positive breast cancer, optimum scheduling of ET should be specified. ${ }^{11}$ 
Clinical studies have demonstrated that fulvestrant shows encouraging efficacy in HER2 positive breast cancer. ${ }^{12,13}$ In a retrospective study, fulvestrant retained activity in HER2 positive breast cancer patients pretreated with trastuzumab. ${ }^{14}$ Fulvestrant is a pure ER antagonist, binding to ER causes its complete blockade: receptor dimerization is impaired, nuclear localization is disrupted, and the bound receptor is rapidly degraded. ${ }^{2,12,13}$ Due to these unique features, fulvestrant blocks the nuclear ER as well as cytoplasmic and membrane-bound ER which are considered to be responsible for ER and HER2 cross-talk.

The current case series reports three patients diagnosed with ER, progesterone receptor (PgR), and HER2 positive breast cancer with metastases at $\geq 2$ sites who were treated with fulvestrant for $\geq 12$ months after failure of anti-HER2 regimens and ET.

\section{Informed consent}

Written informed consent was obtained from the patients for publication of these case reports and accompanying images. Consent was obtained using the standardized informed consent forms of our institution.

\section{Case I}

In October 2009, inflammatory breast cancer with small cutaneous nodules and lymphadenopathy in the axillary and supraclavicular regions was diagnosed in a 63-year-old woman. Histopathological examination proved ER/PgR (Allred scores 7/7) and HER2 (IHC 3+, FISH+) positive adenocarcinoma. Thoracic CT revealed further pathologically enlarged lymph nodes around the brachiocephalic artery and aortic arch. No other metastatic manifestations were detected.

The patient received three lines of trastuzumab-based chemotherapy, which controlled the disease for 15 months (Figure 1). The first-line therapy with paclitaxel-trastuzumab resulted in partial response, but had to be stopped due to grade 3 sensory neuropathy. After two cycles of maintenance trastuzumab monotherapy, the skin metastases rapidly progressed, thus the treatment was supplemented with docetaxel. After sensory neuropathy deteriorated, docetaxel was immediately switched to letrozole. Then, a further three lines of chemotherapy, palliative irradiation to the right breast, chest wall and lymphatic regions, and an additional two lines of HER2-targeted therapy (lapatinib and trastuzumab-emtansine [T-DM1]) were given. These all produced limited or no effect, the disease progressed and involved $>50 \%$ of the chest wall resulting in the typical appearance of "carcinoma en cuirasse". Having no additional anti-HER2 therapy options, fulvestrant (loading and then high-dose schedule with 500 mg every 14/28 days) was started. All cancer manifestations improved, and an almost complete regression was maintained for 2 years. After that, the introduction of letrozole had no effect, while consequently cyclophosphamide/ methotrexate/5-fluorouracil chemotherapy resulted in partial regression which lasted for 8 months. Finally, she died in December 2016 (Figure 1).

\section{Case 2}

In July 2010, a 41-year-old premenopausal woman with shortness of breath and low back pain was diagnosed with metastatic breast cancer. For the past year she had noticed deformation of the right breast. Imaging studies indicated locoregionally advanced disease with distant metastases in the lymph nodes, liver, and bones. Core biopsy from the breast tumor indicated grade 2 invasive ductal carcinoma, ER/PgR (Allred scores 8/4) and HER2 (IHC 3+, FISH+) positive.

As first-line therapy, paclitaxel-trastuzumab followed by trastuzumab maintenance therapy was given, which resulted in major partial regression at all metastatic sites, lasting 19 months. Then the patient developed dizziness and vomiting, and in addition to skeletal progression, multiple brain and leptomeningeal metastases were revealed. After whole-brain irradiation with simultaneous integrated boost $(15 \times 2.2+0.7 \mathrm{~Gy})$ the central nervous system (CNS) metastases completely disappeared.

Following radiotherapy, the patient received eight cycles of cyclophosphamide-epirubicine-5-fluorouracil (CEF), followed by tamoxifen as maintenance therapy. This resulted in stable disease for 16 months altogether. Then, CNS progression was detected, and the patient (with postmenopausal hormone levels) was given fulvestrant (loading and then high-dose schedule, $500 \mathrm{mg}$ every 14/28 days). During the treatment, the lesions in the brain decreased significantly (Figure 2), and all the other manifestations were stable. After 1 year of fulvestrant treatment, the disease progressed in the brain, which was re-irradiated after which T-DM1 treatment was started. As a result, complete regression of the brain metastases associated with partial regression of the other manifestations was observed. The administration of T-DM1 for almost 1 year was subsequently followed by letrozole, which showed no activity. Therapy was continued with capecitabine plus lapatinib, but brain metastases still progressed. Almost 7 years after the first diagnosis, the patient died in January 2017 (Figure 1).

\section{Case 3}

In January 2012, a 50-year-old postmenopausal woman was referred to our department, for the past 2 years she had noticed 


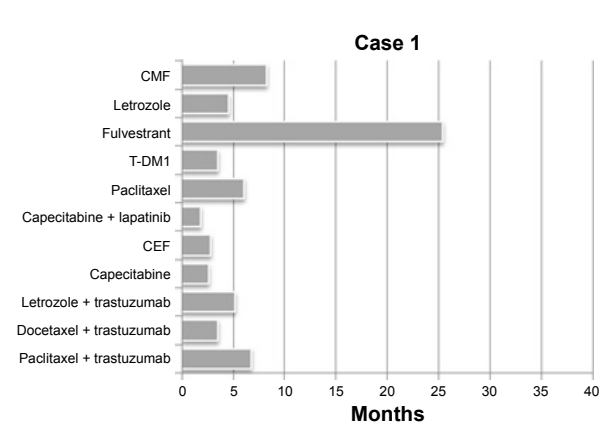

\begin{tabular}{|c|c|c|c|c|}
\hline \multicolumn{2}{|r|}{ Therapy lines } & \multirow{2}{*}{$\begin{array}{l}\text { Best response } \\
P R\end{array}$} & \multirow{2}{*}{$\begin{array}{l}\text { Localization of metastasis } \\
\text { at initiation of therapy } \\
\text { Lymph nodes, skin }\end{array}$} & \multirow{2}{*}{$\begin{array}{l}\text { Reason for therapy discontinuation } \\
\text { Side effect }\end{array}$} \\
\hline 11. & CMF & & & \\
\hline 10. & Letrozole & PD & Lymph nodes, skin & Progression in all Iocalizations \\
\hline 9. & Fulvestrant & PR & Lymph nodes, skin & Progression of skin metastases \\
\hline 8. & T-DM1 & PD & Lymph nodes, skin & Progression of skin metastases \\
\hline 7. & $\begin{array}{l}\text { Paclitiaxel } \\
\end{array}$ & SD & Lymph nodes, skin & Progression in all localizations \\
\hline 6. & Capecitabine + lapatinib & PD & Lymph nodes, skin & Progression in all Iocalizations \\
\hline 5. & CEF & SD & Lymph nodes, skin & Progression of $1 \mathrm{ymph}$ node metastases \\
\hline 4. & Capecitabine & PD & Lymph nodes, skin & Progression in all localizations \\
\hline 3. & Letrozole + trastuzumab & SD & Lymph nodes, skin & Progression in all localizations \\
\hline 2. & Docetaxel + trastuzumab & SD & Lymph nodes, skin & Side effect \\
\hline \multirow{2}{*}{1.} & Trastuzumab monotherapy & PD & $\begin{array}{l}\text { Lymph nodes, skin involvement } \\
\text { is suspected }\end{array}$ & $\begin{array}{l}\text { Progression of skin and lymph node } \\
\text { metastases }\end{array}$ \\
\hline & Paclitaxel + trastuzumab & $P R$ & $\begin{array}{l}\text { Lymph nodes, skin involvement } \\
\text { is suspected }\end{array}$ & Side effect \\
\hline
\end{tabular}

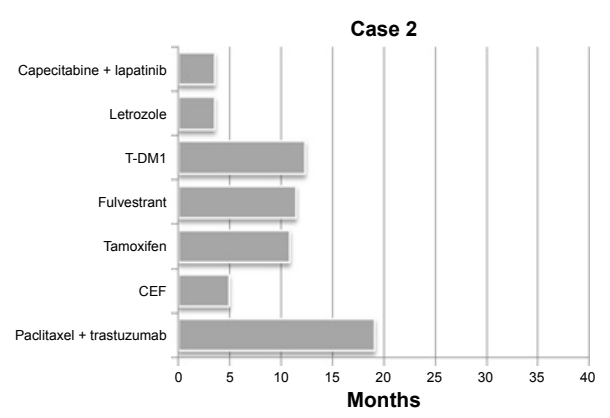

\begin{tabular}{|c|c|c|c|c|}
\hline \multicolumn{2}{|r|}{ Therapy lines } & \multirow{2}{*}{$\begin{array}{l}\text { Best response } \\
\text { PD }\end{array}$} & \multirow{2}{*}{$\begin{array}{l}\begin{array}{l}\text { Localization of metastasis } \\
\text { at initiation of therapy }\end{array} \\
\text { Lymph nodes, liver, bone, brain }\end{array}$} & \multirow{2}{*}{$\begin{array}{l}\text { Reason for therapy discontinuation } \\
\text { Progression of brain metastases }\end{array}$} \\
\hline 7. & Capecitabine + lapatinib & & & \\
\hline 6. & Letrozole & PD & Lymph nodes, liver, bone, brain & Progression of brain and bone metastases \\
\hline 5. & T-DM1 & PR & Lymph nodes, liver, bone, brain & Progression of brain metastases \\
\hline 4. & Fulvestrant & PR & Lymph nodes, liver, bone, brain & Progression of brain metastases \\
\hline 3. & Tamoxifen & SD & Lymph nodes, liver, bone, brain & Progression of brain metastases \\
\hline 2. & CEF & SD & Lymph nodes, liver, bone, brain & Maximum dose of anthracycline \\
\hline \multirow{2}{*}{1.} & Trastuzumab monotherapy & PR & Lymph nodes, liver, bone & Brain metastasis (progression) \\
\hline & Paclitaxel + trastuzumab & PR & Lymph nodes, liver, bone & \\
\hline
\end{tabular}

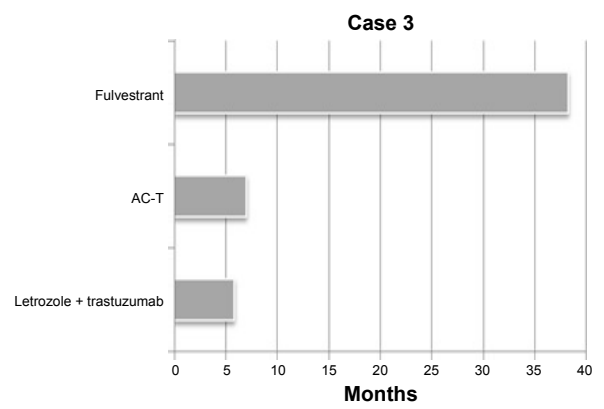

\begin{tabular}{|c|l|l|l|l|}
\hline \multicolumn{2}{|c|}{ Therapy lines } & Best response & $\begin{array}{l}\text { Localization of metastasis } \\
\text { at intitition of therapy }\end{array}$ & Reason for therapy discontinuation \\
\hline \multirow{2}{*}{3.} & Fulvestrant reinduction & SD & Bone, brain & Liver and spleen metastases (progression) \\
\cline { 2 - 5 } & Fulvestrant & PR & Bone & Brain metastasis (progression) \\
\hline \multirow{2}{*}{2.} & $\begin{array}{l}\text { 4xAC followed by } \\
\text { weekky pacitiaxel }\end{array}$ & PR & Bone & Side effect \\
\hline 1. & Letrozole + trastuzumab & PD & Bone & Progression of bone metastases \\
\hline
\end{tabular}

Figure I Treatment lines, durations, and outcomes of Cases I-3.

Abbreviations: AC-T, doxorubicin-cyclophosphamide and sequential paclitaxel; CEF, cyclophosphamide-epirubicine-5-fluorouracil; CMF, cyclophosphamide-methotrexate5-fluorouracil; PD, progressive disease; PR, partial response; SD, stable disease; T-DMI, trastuzumab-emtansine.
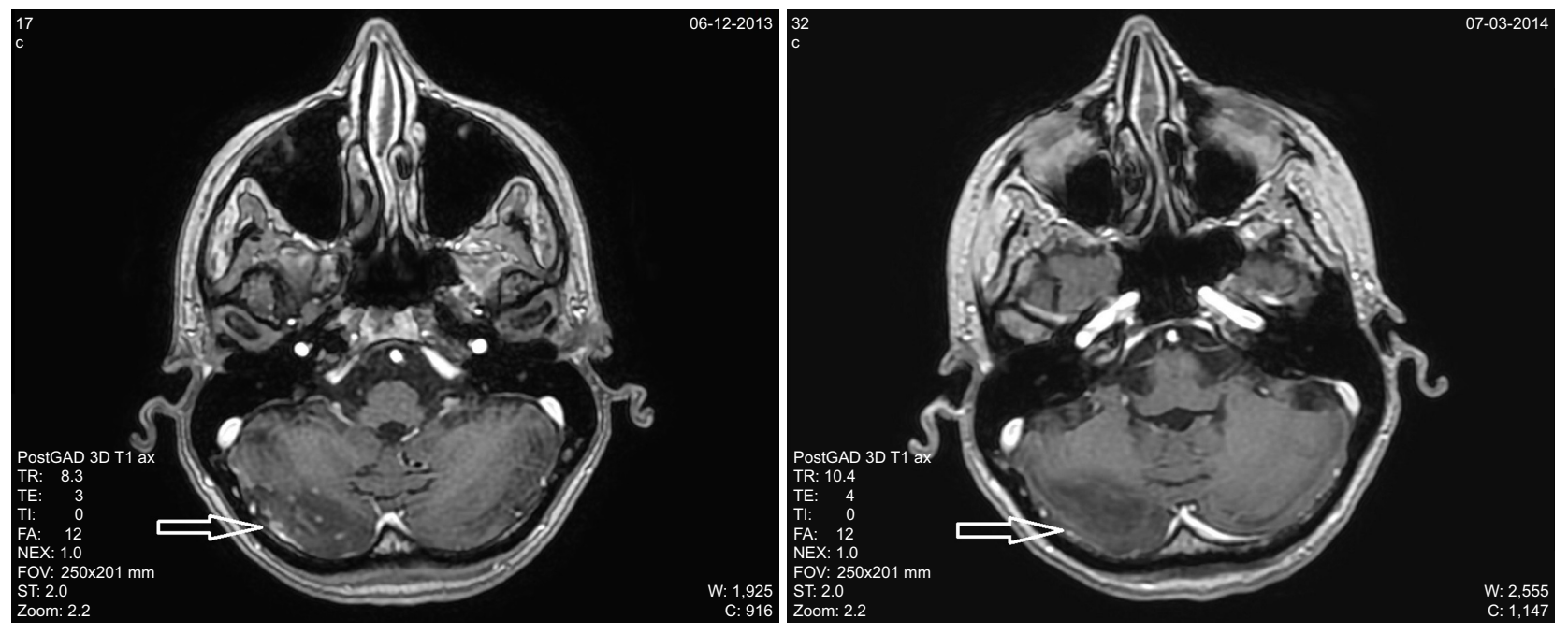

Figure 2 Brain metastases before and 3 months after the introduction of fulvestrant therapy (Case 2). 
a palpable node in her right breast. Extensive stage T3N3 breast cancer with multiple bone metastases was diagnosed. Histopathological examination revealed grade 3 invasive ductal cancer, ER/PgR (Allred scores 8/8) and HER2 IHC 3+ positive. As first-line treatment, letrozole plus trastuzumab was initiated. During treatment, the tumor markers (CA15-3 and CEA) increased and 5 months later skeletal progression was detected. Then, following four courses of doxorubicin plus cyclophosphamide, paclitaxel (once every 3 weeks) was initiated. Tumor markers decreased to normal levels and partial response was detected at all metastatic sites, however, due to grade 3 peripheral neuropathy, paclitaxel treatment was terminated after 18 cycles. Then, fulvestrant (loading and then high-dose schedule, $500 \mathrm{mg}$ every 14/28 days) was introduced as maintenance therapy. After 19 months of fulvestrant therapy, the patient presented with CNS symptoms; brain CT verified multiple metastatic lesions in the cerebellum and parieto-occipital region. Whole brain irradiation with simultaneous integrated boost (14×2.2 Gy+14×0.8 Gy) was applied. Since all the extracranial tumor manifestations were controlled with fulvestrant, the patient remained on the systemic therapy; following another 14 months of fulvestrant therapy, no progression was detected until June 2017. At that time, although the brain metastases remained stable, new metastases in the liver and spleen, as well as progression of the bone metastases were diagnosed. The patient's general condition deteriorated continuously and she died in September 2017 (Figure 1).

\section{Discussion}

We reported the durable effect of fulvestrant monotherapy in three metastatic ER and HER2 positive breast cancer patients. The common features among the cases were pretreatment with and acquired resistance to trastuzumab; fulvestrant controlled disease manifestations (extensive skin involvement and CNS metastases) which, according to guidelines, are a priori considered hormone-resistant and are primarily treated with chemotherapy.

Our experience is consistent with the knowledge that in luminal B-type breast cancers (including HER2 and HR positive tumors), a vulnerable balance exists between ER-directed and growth factor-directed driver mechanisms. HER2 blockade induces dependence on HR signaling which serves as an escape function, a probably frequent resistance mechanism to anti-HER2 agents in HR positive cancers. ${ }^{3,4}$ Indeed, HER2 blockade may induce the expression of ER, even in originally ER negative breast cancers, as demonstrated in the study of Giuliano et al. ${ }^{4}$ The sequential use of HER2 blockade and ER inhibition targets the actual escape mechanism evolved due to the cross-talk between the two driver pathways. Limited clinical data exist on the significance of alternating driver mechanisms in this patient population. Robertson et al reported the largest study about the activity of fulvestrant in HER2 positive breast cancer. In 102 heavily pretreated patients, the median duration of treatment was 14.5 months. The clinical benefit rate in the overall population was $42 \%$, which varied according to whether pretreatment with trastuzumab was (46.5\%) or was not $(39 \%)$ present. Among those who responded to prior trastuzumab, the clinical benefit rate was $50 \% .{ }^{14}$ Notably, while in this pooled analysis low-dose fulvestrant therapy of $250 \mathrm{mg} / \mathrm{month}$ was reported, our patients received the loading and high-dose schedule.

T-DM1 is an antibody drug conjugate that is active in advanced HER2 positive breast cancer after trastuzumab therapy. Out of our two cases who received T-DM1, the patient who responded to the T-DM1 received fulvestrant prior to it; while the other patient, who had been treated with a series of chemotherapies with or without trastuzumab, but not with mono ET, showed resistance to T-DM1. In fact, the presence of HER2 in cancer cells is essential for T-DM1 efficacy and cases with higher HER2 mRNA levels respond more often to T-DM1 than those with lower HER2 expression. ${ }^{15}$ Estrogen deprivation with $\mathrm{AI}$ or fulvestrant results in the upregulation of HER2 in HER2 and HR positive tumors. ${ }^{2-4,16-18}$ Based on the preclinical and clinical findings that tumors can alternate between the dominance of ER-driven or HER2-driven pathways, ${ }^{2,4,17,18}$ the question arises whether the use of ET before T-DM1 or use of ET between two lines of anti-HER2 treatments may increase the benefits of therapy regimens. Moreover, this therapy sequence favors a lower incidence of anti-HER 2 therapy-related cardiac side effects.

We found it very intriguing that fulvestrant was active in disease entities such as extensive skin involvement or CNS metastasis traditionally not treated with ET. Actually, fulvestrant does not cross the intact blood-brain barrier and knowledge about its efficacy in brain metastasis is limited. ${ }^{19}$

Recently, dual HER2 blockade with trastuzumab and lapatinib combined with AI in the first-line setting was shown to improve efficacy in the ALTERNATIVE study, by means of prolonged progression-free survival, higher response rate, and a trend of superior overall survival. ${ }^{20}$ The strategy of combining multiple targeted agents is aimed at the prevention or blockade of different resistance mechanisms. The combination of new targeted therapies, such as the CDK4/6 inhibitors or the PI3K/mTOR inhibitors with 
endocrine and/or anti-HER2 therapies may not only improve outcomes, but could serve as a preferable alternative to chemotherapy. ${ }^{21,22}$

\section{Conclusion}

ET is an essential component in the multimodal management of advanced HER2 and HR positive breast cancer. The exact place where endocrine agents fit into multimodal management should be explored based on further clinical trials. According to our limited experience, fulvestrant is an especially attractive treatment option in metastatic HER2 positive breast cancer after progression on previous HER2targeted treatments.

\section{Acknowledgment}

This research did not receive any specific grant from funding agencies in the public, commercial, or not-for-profit sectors.

\section{Disclosure}

The authors report no conflicts of interest in this work.

\section{References}

1. Loi S, Dafni U, Karlis D, et al. Effects of Estrogen Receptor and Human Epidermal Growth Factor Receptor-2 Levels on the Efficacy of Trastuzumab: A Secondary Analysis of the HERA Trial. JAMA Oncol. 2016;2(8):1040-1047.

2. Osborne CK, Schiff R. Mechanisms of endocrine resistance in breast cancer. Аnпи Rev Med. 2011;62:233-247.

3. Menyhárt O, Santarpia L, Győrffy B. A Comprehensive Outline of Trastuzumab Resistance Biomarkers in HER2 Overexpressing Breast Cancer. Curr Cancer Drug Targets. 2015;15(8):665-683.

4. Giuliano M, Hu H, Wang YC, et al. Upregulation of ER Signaling as an Adaptive Mechanism of Cell Survival in HER2-Positive Breast Tumors Treated with Anti-HER2 Therapy. Clin Cancer Res. 2015; 214003(17):3995-4003.

5. Montemurro F, di Cosimo S, Arpino G. Human epidermal growth factor receptor 2 (HER2)-positive and hormone receptor-positive breast cancer: new insights into molecular interactions and clinical implications. Ann Oncol. 2013;24(11):2715-2724.

6. Schwarzberg LS, Franco SX, Florance A, et al. Lapatinib plus letrozole as first-line therapy for HER-2+ hormone receptor-positive metastatic breast cancer. Oncologist. 2010;15(2):122-129.

7. Johnston S, Pippen J, Pivot X, et al. Lapatinib combined with letrozole versus letrozole and placebo as first-line therapy for postmenopausal hormone receptor-positive metastatic breast cancer. J Clin Oncol. 2009;27(33):5538-5546.

OncoTargets and Therapy

\section{Publish your work in this journal}

OncoTargets and Therapy is an international, peer-reviewed, open access journal focusing on the pathological basis of all cancers, potential targets for therapy and treatment protocols employed to improve the management of cancer patients. The journal also focuses on the impact of management programs and new therapeutic agents and protocols on
8. Huober J, Fasching PA, Barsoum M, et al. Higher efficacy of letrozole in combination with trastuzumab compared to letrozole monotherapy as first-line treatment in patients with HER2-positive, hormone-receptorpositive metastatic breast cancer - results of the eLEcTRA trial. Breast. 2012;21(1):27-33

9. Kaufman B, Mackey JR, Clemens MR, et al. Trastuzumab plus anastrozole versus anastrozole alone for the treatment of postmenopausal women with human epidermal growth factor receptor 2-positive, hormone receptor-positive metastatic breast cancer: results from the randomized phase III TAnDEM study. J Clin Oncol. 2009;27(33): 5529-5537.

10. Giordano SH, Temin S, Kirshner JJ, et al. Systemic therapy for patients with advanced human epidermal growth factor receptor 2-positive breast cancer: American Society of Clinical Oncology clinical practice guideline. J Clin Oncol. 2014;32(19):2078-2099.

11. Rugo HS, Rumble RB, Macrae E, et al. Endocrine Therapy for Hormone Receptor-Positive Metastatic Breast Cancer: American Society of Clinical Oncology Guideline. J Clin Oncol. 2016;34(25):3069-3103.

12. Steger GG, Gips M, Simon SD, et al. Fulvestrant ("Faslodex"): clinical experience from the Compassionate Use Programme. Cancer Treat Rev. 2005;31 Suppl 2:S10-S16.

13. Steger GG, Bartsch R, Wenzel C, et al. Fulvestrant ('Faslodex') in pretreated patients with advanced breast cancer: a single-centre experience. Eur J Cancer. 2005;41(17):2655-2661.

14. Robertson JF, Steger GG, Neven P, et al. Activity of fulvestrant in HER2-overexpressing advanced breast cancer. Ann Oncol. 2010; 21(6):1246-1253.

15. Barok M, Joensuu H, Isola J. Trastuzumab emtansine: mechanisms of action and drug resistance. Breast Cancer Res. 2014;16(2):209.

16. Massarweh S, Osborne CK, Jiang S, et al. Mechanisms of tumor regression and resistance to estrogen deprivation and fulvestrant in a model of estrogen receptor-positive, HER-2/neu-positive breast cancer. Cancer Res. 2006;66(16):8266-8273.

17. Munzone E, Curigliano G, Rocca A, et al. Reverting estrogen-receptornegative phenotype in HER-2-overexpressing advanced breast cancer patients exposed to trastuzumab plus chemotherapy. Breast Cancer Res. 2006;8(1):R4.

18. Xia W, Bacus S, Hegde P, et al. A model of acquired autoresistance to a potent ErbB2 tyrosine kinase inhibitor and a therapeutic strategy to prevent its onset in breast cancer. Proc Natl Acad Sci U S A. 2006; 103(20):7795-7800.

19. Howell A, Osborne CK, Morris C, Wakeling AE, Ici WAE. ICI 182,780 (Faslodex): development of a novel, "pure" antiestrogen. Cancer. 2000; 89(4):817-825.

20. Johnston SRD, Hegg R, Im SA, et al. Phase III, Randomized Study of Dual Human Epidermal Growth Factor Receptor 2 (HER2) Blockade With Lapatinib Plus Trastuzumab in Combination With an Aromatase Inhibitor in Postmenopausal Women With HER2-Positive, Hormone Receptor-Positive Metastatic Breast Cancer: ALTERNATIVE. J Clin Oncol. 2018;36(8):741-748.

21. Gianni L, Bisagni G, Colleoni M, et al. Neoadjuvant treatment with trastuzumab and pertuzumab plus palbociclib and fulvestrant in HER2positive, ER-positive breast cancer (NA-PHER2): an exploratory, open-label, phase 2 study. Lancet Oncol. 2018;19(2):249-256.

22. Corona SP, Ravelli A, Cretella D, et al. CDK4/6 inhibitors in HER2positive breast cancer. Crit Rev Oncol Hematol. 2017;112:208-214.

\section{Dovepress}

patient perspectives such as quality of life, adherence and satisfaction. The manuscript management system is completely online and includes a very quick and fair peer-review system, which is all easy to use. Visit http://www.dovepress.com/testimonials.php to read real quotes from published authors. 